\title{
Poisson Equations with Nonlinear Source Terms on Networks
}

\author{
By \\ Soon-Yeong Chung, Dohan KIM and Nam Kee LeE**
}

\begin{abstract}
C. Berenstein and the first author introduced an elliptic operator $\Delta_{\omega}$ and an $\omega$ harmonic function on graphs, with its physical interpretation as a diffusion equation on graphs, which models electric networks. They also proved the solvability of the problems such as the Dirichlet and Neumann boundary value problems for the Laplace equation and the Poisson equation on networks. In this paper, we consider the Poisson equation with nonlinear source term on networks and show the existence and the uniqueness of a solution with suitable source term.
\end{abstract}

\section{$\S 1 . \quad$ Introduction}

A network represents a way of interconnecting any pair of users or nodes by means of some meaningful links. Thus, it is quite natural that its structure can be represented, at least in a simplified form, by a connected graph whose vertices represent nodes and whose edges represent their links.

In [1] the authors introduce an elliptic operator on the graph, the $\omega$ Laplacian $\Delta_{\omega}$ and interpret it as a diffusion equation on the graph modeled

Communicated by H. Okamoto. Received August 29, 2006. Revised December 12, 2006. 2000 Mathematics Subject Classification(s): 35J65, 94C15.

Key words: discrete Laplacian, Poisson equation.

The first author was supported by Korea Research Foundation Grant. KRF-2003-041C00032 and the Special Grant of Sogang University in 2006.

The second author was supported by Korea Research Foundation Grant. KRF-2005-015C00019.

*Department of Mathematics and Program of Integrated Biotechnology, Sogang University, Seoul 121-742, Korea.

e-mail: sychung@sogang.ac.kr

** Department of Mathematics, Seoul National University, Seoul 151-747, Korea.

e-mail: dhkim@snu.ac.kr and paulee74@math.snu.ac.kr 
by the electric network. Using tools of partial differential equations, they gave some results on direct and inverse problems about Laplacian equation and linear Poisson equation.

The $\omega$-Laplacian $\Delta_{\omega}$ of a function $u: V(G) \rightarrow \mathbf{R}$ on a weighted graph $G$ is defined by

$$
\Delta_{\omega} u(x):=\sum_{y \in V(G)}[u(y)-u(x)] \cdot \frac{\omega(x, y)}{d_{\omega} x}, \quad x \in V(G),
$$

where $\omega(x, y)$ is the weight on the edge connecting vertices $x$ and $y$, and $d_{\omega} x=$ $\sum_{y \sim x} \omega(x, y)$.

It can be interpreted as a diffusion equation on the graph modeled by the electric network.

They also defined the directional derivatives $D_{\omega, y}$ and the gradient vector $\nabla_{\omega}$ on networks and considered the $\omega$-Laplacian $\Delta_{\omega}$ as following:

$$
\Delta_{\omega} u(x)=-\sum_{y \in S}\left(D_{\omega, y}^{2} u\right)(x), \quad x \in V(G) .
$$

By the above interpretation we can consider the $\omega$-Laplacian $\Delta_{\omega}$ as an elliptic operator on networks so we can apply the tools of partial differential equation to the network problems. For example, the following Dirichlet boundary value problem for the Poisson equation on networks was solved.

Theorem 1.1. $\quad$ Let $S$ be a subgraph of a host graph with a weight $\omega$ and $\sigma: \partial S \rightarrow \mathbf{R}$ be a given function. Then the unique solution $u$ to the Dirichlet boundary value problem (DBVP)

$$
\left\{\begin{array}{l}
\Delta_{\omega} u(x)=g(x), \quad x \in S \\
\left.u\right|_{\partial S}=\sigma
\end{array}\right.
$$

can be represented as

$$
u(x)=-\left\langle\gamma_{\omega, S}(x, \cdot), B_{\sigma}\right\rangle_{S}+\left\langle\gamma_{\omega, S}(x, \cdot), g\right\rangle_{S},
$$

where $\gamma_{\omega, S}(x, y)$ is discrete Green function on $S$ and

$$
B_{\sigma}(y)=\sum_{z \in \partial S} \frac{\sigma(z) \omega(y, z)}{d_{\omega} y}, \quad y \in S .
$$


Physically we may consider the function $g$ as an external current source in the electric networks. Since $g$ does not depend on $u,(1.2)$ is nonhomogeneous and linear problem.

In [9] and [10] the authors considered the nonlinear Laplace operator on networks, i.e.

$$
\Delta_{\omega, p} u(x):=\sum_{y \in S}|u(y)-u(x)|^{p-2}(u(y)-u(x)) \cdot \frac{\omega(x, y)}{d_{\omega} x}, \quad x \in S .
$$

They also solved the existence of a solution of the following Nonlinear Poisson equation:

$$
\Delta_{\omega, p} u(x)=g(x) \quad \text { on } S .
$$

In this paper we study nonlinear Poisson equation on network $\bar{S}$. But the operator is linear and the nonlinearity comes from the source term. Let $u: \bar{S} \rightarrow \mathbf{R}, f: \bar{S} \times \mathbf{R} \rightarrow \mathbf{R}$ and $\sigma: \partial S \rightarrow \mathbf{R}$.

$$
\left\{\begin{array}{l}
-\Delta_{\omega} u(x)=f(x, u(x)), \quad x \in S, \\
u(x)=\sigma(x), \quad x \in \partial S
\end{array}\right.
$$

with some conditions on $f$ and $\sigma$.

We will deal with two cases.

Case I : Suppose that $\sigma \equiv 0$ and $f(x, u(x))=f(u(x))$ is smooth and, for some $p>1$, satisfies following conditions for every $s \in \mathbf{R}$

$$
|f(s)| \leq C\left(1+|s|^{p}\right), \quad\left|f^{\prime}(s)\right| \leq C\left(1+|s|^{p-1}\right),
$$

where $C$ is a constant. We suppose also that for constants $0<\alpha \leq \beta$

$$
\alpha|s|^{p+1} \leq F(s) \leq \beta|s|^{p+1},
$$

where $F(s):=\int_{0}^{s} f(r) d r$.

Case II : Suppose that the function $f$ is bounded and nonincreasing with respect to the second variable.

(1.7) implies that $f$ in the Case I is increasing near the origin and unbounded, so that the Case I and II are different problems. 
We organized this paper as follows: In Section 2, we prepare some calculus on graph which are given in [1]. We define directional derivatives of the functions on the network and understand $\omega$ - Laplacian as a sum of second derivatives of all direction. And we show the good properties of $\omega$ - Laplacian such as Green's formula.

In Section 3, we will show the the existence of nonzero solution for Case I. To show it we need Mountain Pass Theorem for nonlinear functional.

In Section 4, we show the existence of solution for Case II of (1.6) by Schaefer's fixed point theorem and the uniqueness by maximum principle.

\section{$\S 2 . \quad$ Preliminaries}

We shall begin with some definitions of graph theoretic notions frequently used throughout this paper.

By a graph $G=G(V, E)$ we mean a finite set $V$ of vertices with a set $E$ of two-element subset of $V$ (whose elements are called edges). The set of vertices and edges of a graph $G$ are sometimes denoted by $V(G)$ and $E(G)$, or $V$ and $E$ simply, respectively. But conventionally, we mean by $x \in V$, and $x \in G$ that $x$ is a vertex in $\mathrm{G}$.

A graph $S=S\left(V^{\prime}, E^{\prime}\right)$ is said to be a subgraph of $G(V, E)$ if $V^{\prime} \subset V$ and $E^{\prime} \subset E$. If $E^{\prime}$ consists of all the edges from $E$ which connect the vertices in $V^{\prime}$, then $S$ is called an induced subgraph.

A weighted (undirected) graph is a graph $G(V, E)$ associated with a weight function $\omega: V \times V \rightarrow[0, \infty)$ satisfying

(i) $\omega(x, x)=0, \quad x \in V$,

(ii) $\omega(x, y)=\omega(y, x), \quad$ if $x \sim y$,

(iii) $\omega(x, y)=0, \quad$ if $\{x, y\} \notin E$.

Here, $\{x, y\}$ denotes the edge connecting the vertices $x, y$ and $x \sim y$ means that two vertices $x$ and $y$ are connected (adjacent) by an edge in $E$.

In particular, a weight function $\omega$ satisfying

$$
\omega(x, y)=1, \quad \text { if } x \sim y
$$

is called a standard weight on $G$.

The degree $d_{\omega} x$ of a vertex $x$ in a weighted graph $G(V, E)$ with a weight $\omega$ is defined to be

$$
d_{\omega} x:=\sum_{y \in V} \omega(x, y)
$$


A graph $G$ is said to be connected if for every vertices $x$ and $y$ there exist a sequence (termed a path or trail) of vertices $x=x_{0}, x_{1}, x_{2}, \ldots, x_{n-1}, x_{n}=y$ such that $x_{j-1} \sim x_{j}, j=1,2, \ldots, n$. Then it is easy to see that every induced subgraph of a connected graph is also connected.

Throughout this paper, all the subgraphs in our concern are assumed to be induced subgraphs of a host connected graph with a weight and a function on a graph is understood as a function defined only on the set of vertices.

The integration of a function $u: G \rightarrow \mathbf{R}$ on a graph $G=G(V, E)$ is defined to be

$$
\int_{G} u d_{\omega} \quad\left(\operatorname{or} \int_{G} u(x) d_{\omega} x\right):=\sum_{x \in V} u(x) d_{\omega} x .
$$

We shall now define a directional derivative of a function $u: G \rightarrow \mathbf{R}$. For $x$ and $y \in V$ we define

$$
D_{\omega, y} u(x):=[u(y)-u(x)] \sqrt{\frac{\omega(x, y)}{d_{\omega} x}} .
$$

The gradient $\nabla_{\omega}$ of function $u$ is defined to be a vector

$$
\nabla_{\omega} u(x):=\left(D_{\omega, y} u(x)\right)_{y \in V} .
$$

Then it is easy to see that

$$
\begin{aligned}
\left|\nabla_{\omega} u(x)\right|^{2} & =\sum_{y \in V}\left|D_{\omega, y} u(x)\right|^{2} \\
& =\sum_{y \in V}|u(y)-u(x)|^{2} \cdot \frac{\omega(x, y)}{d_{\omega} x}
\end{aligned}
$$

and

$$
\begin{aligned}
\int_{G}\left|\nabla_{\omega} u(x)\right|^{2} d_{\omega} x & =\sum_{x \in V}\left|\nabla_{\omega} u(x)\right|^{2} d_{\omega} x \\
& =\sum_{x \in V} \sum_{y \in V}|u(y)-u(x)|^{2} \omega(x, y) \\
& =2 \sum_{\{x, y\} \in E}|u(y)-u(x)|^{2} \omega(x, y) .
\end{aligned}
$$

For a subgraph $S$ of a graph $G=G(V, E)$ the (vertex) boundary $\partial S$ of $S$ is defined by the set of all vertices $z \in V$ not in $S$ but adjacent to some vertex in $S$, i.e.

$$
\partial S:=\{z \in V \backslash S \mid z \sim y \text { for some } y \in S\}
$$


and by $\bar{S}$ we denote a graph whose vertices and edges are in $S \cup \partial S$.

The (outward) normal derivative $\frac{\partial u}{\partial_{\omega} n}(z)$ at $z \in \partial S$ is defined to be

$$
\frac{\partial u}{\partial_{\omega} n}(z):=\sum_{y \in S}[u(z)-u(y)] \cdot \frac{\omega(z, y)}{d_{\omega}^{\prime} z},
$$

where $d_{\omega}^{\prime} z=\sum_{y \in S} \omega(z, y)$.

Now, we can write the $\omega$-Laplacian $\Delta_{\omega}$ of a function $u: G \rightarrow \mathbf{R}$ on a graph $G$ using directional derivatives,

$$
\Delta_{\omega} u(x)=\sum[u(y)-u(x)] \cdot \frac{\omega(x, y)}{d_{\omega} x}=-\sum_{y \in V}\left(D_{y}^{2} u\right)(x), \quad x \in V .
$$

In what follows, a function $u$ defined on $\bar{S}$ may be understood as a function on its host graph $G$ so that $u=0$ on $G \backslash \bar{S}$, if necessary. Also, we assume that each $z_{1}, z_{2} \in \partial S$ are not directly connected by an edge. (By these assumptions, the symbol $d_{\omega}^{\prime} z$ may be replaced by $d_{\omega} z$.)

Theorem 2.1 [1]. Let $S$ be a subgraph of a host graph $G$. Then for functions $u: \bar{S} \rightarrow \mathbf{R}$ and $v: \bar{S} \rightarrow \mathbf{R}$, we have

$$
2 \int_{\bar{S}} v\left(-\Delta_{\omega} u\right) d_{\omega}=\int_{\bar{S}} \nabla_{\omega} v \cdot \nabla_{\omega} u d_{\omega} .
$$

Corollary 2.1 [1]. $\quad$ Under the same hypothesis as above we have

(i)

$$
2 \int_{\bar{S}} f\left(-\Delta_{\omega} f\right) d_{\omega}=\int_{\bar{S}}\left|\nabla_{\omega} f\right|^{2} d_{\omega}
$$

(ii)

$$
\int_{\bar{S}} h \Delta_{\omega} f d_{\omega}=\int_{\bar{S}} f \Delta_{\omega} h d_{\omega},
$$

(iii) (Green's formula)

$$
\int_{S}\left(f \Delta_{\omega} h-h \Delta_{\omega} f\right) d_{\omega}=\int_{\partial S}\left(f \frac{\partial h}{\partial_{\omega} n}-h \frac{\partial f}{\partial_{\omega} n}\right) d_{\omega} .
$$


In the continuous case, the followings are well-known formula :

$$
\begin{aligned}
& \Delta(f g)=f \Delta g+2 \nabla f \cdot \nabla g+g \Delta f \\
& \int_{\Omega} \nabla f \cdot \nabla g+\int_{\Omega} f \Delta g=\int_{\partial \Omega} f \frac{\partial g}{\partial n} .
\end{aligned}
$$

Here, we introduce a discrete analogue of them.

Theorem 2.2 [1]. Under the same hypothesis as in Theorem 2.1 we have followings :

(i)

$$
\Delta_{\omega}(f h)=f \Delta_{\omega} h+\nabla_{\omega} f \cdot \nabla_{\omega} h+h \Delta_{\omega} f
$$

(ii)

$$
\int_{S} \nabla_{\omega} f \cdot \nabla_{\omega} h d_{\omega}+\int_{S}\left[f \Delta_{\omega} h+h \Delta_{\omega} f\right] d_{\omega}=\int_{\partial S} \frac{\partial(f h)}{\partial_{\omega} n} d_{\omega}
$$

From now on, if there is no risk of confusion we use the notation $\nabla$ and $\Delta$ instead of using $\nabla_{\omega}$ and $\Delta_{\omega}$ briefly.

We also consider the function space on network. Let $X=\{u \mid u: \bar{S} \rightarrow \mathbf{R}\}$ be a set of functions defined on finite network. Then for $1<p \leq \infty$, we can consider the usual $L^{p}=\left(X,\|\cdot\|_{p}\right)$ space with $L^{p}$ norm on $X$,

$$
\|v\|_{p}^{p}=\int_{\bar{S}}|v(x)|^{p} d_{\omega} x, \quad \text { for } 1<p<\infty,
$$

and

$$
\|v\|_{\infty}=\max _{x \in \bar{S}}\{|v(x)|\} .
$$

Specially $H=\left(X,\|\cdot\|_{H}\right)$ is a Hilbert space with the inner product

$$
(v, w)_{H}=\frac{1}{2} \int_{\bar{S}} \nabla v(x) \cdot \nabla w(x) d_{\omega} x+\int_{\partial S} v(z) w(z) d_{\omega} z,
$$

and the norm

$$
\|v\|_{H}^{2}=\frac{1}{2} \int_{\bar{S}}|\nabla v(x)|^{2} d_{\omega} x+\int_{\partial S}|v(z)|^{2} d_{\omega} z .
$$

Since the network contains finite nodes and all the norms on the finite dimensional spaces are equivalent, all the norms on the same network are equivalent. 


\section{$\S 3 . \quad$ Existence of Non-trivial Solution Case I}

In this section we will consider the following Dirichlet boundary value problem :

$$
\left\{\begin{array}{l}
-\Delta u(x)=f(u(x)), \quad x \in S, \\
u(x)=0, \quad x \in \partial S .
\end{array}\right.
$$

We assume $f$ is smooth and, for some $p>1$, satisfies following conditions for every $s \in \mathbf{R}$

$$
|f(s)| \leq C\left(1+|s|^{p}\right), \quad\left|f^{\prime}(s)\right| \leq C\left(1+|s|^{p-1}\right),
$$

where $C$ is a constant. We suppose also that for constants $0<\alpha \leq \beta$

$$
\alpha|s|^{p+1} \leq F(s) \leq \beta|s|^{p+1},
$$

where $F(s):=\int_{0}^{s} f(r) d r$.

Now (3.2) implies $f(0)=0$, and so obviously $u \equiv 0$ is a trivial solution of (3.1). We want to find non-trivial solution.

Remark. For $p>1$ the function $f(\xi)=|\xi|^{p-1} \xi$ satisfies the hypotheses. So the following equation is a canonical example of Case $\mathbf{I}$.

$$
\left\{\begin{array}{l}
-\Delta u(x)=|u(x)|^{p-1} u(x), \quad x \in S \\
u(x)=0, \quad x \in \partial S
\end{array}\right.
$$

Let $X_{0}=\{u: \bar{S} \rightarrow \mathbf{R} \mid u(z)=0$ for $z \in \partial S\}$. Then the subspace $H_{0}=\left(X_{0},\|\cdot\|_{H}\right)$ of $H$ is Hilbert space with inner product

$$
(v, w)=\frac{1}{2} \int_{\bar{S}} \nabla v(x) \cdot \nabla w(x) d_{\omega} x,
$$

and norm

$$
\|v\|_{H}^{2}=\frac{1}{2} \int_{\bar{S}}|\nabla v(x)|^{2} d_{\omega} x .
$$

We briefly use the notation $\|v\|$ instead of $\|v\|_{H}$.

To show the existence of nonzero solution by critical point of a nonlinear functional, we need to define the derivative of nonlinear functional on Hilbert space. 
Definition 3.1. A nonlinear functional $I: H_{0} \rightarrow \mathbf{R}$ is differentiable at $u \in H_{0}$ if there exists $v \in H_{0}$ such that

$$
I[w]=I[u]+(v, w-u)+o(\|w-u\|) \quad\left(\forall w \in H_{0}\right) .
$$

The element $v$, if it exists, is unique. We then write $I^{\prime}[u]=v$.

Now we state the Mountain Pass Theorem which guarantees the existence of critical point of functional ([7]).

Theorem 3.1 (Mountain Pass Theorem). Assume that $I^{\prime}[u]$ exist for all $u \in H_{0}$ and is Lipschitz continuous on bounded subsets of $H_{0}$. Suppose also (i) $I[0]=0$,

(ii) there exist constants $r, a>0$ such that

$$
I[u] \geq a \text { if }\|u\|=r
$$

(iii) there exists an element $v \in H_{0}$ with

$$
\|v\|>r, \quad I[v] \leq 0
$$

Define

$$
\Gamma:=\left\{g:[0,1] \rightarrow H_{0} \mid g \text { is continuous, } g(0)=0 \text { and } g(1)=v\right\} .
$$

Then

$$
c=\inf _{g \in \Gamma} \max _{0 \leq t \leq 1} I[g(t)]
$$

is a critical value of $I$, i.e. $\exists u \in H_{0}$ such that $I[u]=c$ and $I^{\prime}[u]=0$.

Now we are ready to state and prove the main result of this section.

Theorem 3.2. $\quad$ The Dirichlet boundary value problem (3.1) has at least one nonzero solution.

Proof. Define a nonlinear functional

$$
I[u]:=\int_{\bar{S}} \frac{1}{4}|\nabla u|^{2}-F(u) d_{\omega} x
$$

for $u \in H_{0}$. We intend to apply the Mountain Pass Theorem to $I[\cdot]$.

$$
I[u]=\frac{1}{2}\|u\|^{2}-\int_{\bar{S}} F(u) d_{\omega}=: I_{1}[u]-I_{2}[u] .
$$


First we have to show that $I$ is differentiable and $I^{\prime}$ is Lipschitz continuous on bounded subsets of $H_{0}$.

To see this, note first that for each $u, w \in H_{0}$,

$$
I_{1}[w]=\frac{1}{2}\|w\|^{2}=\frac{1}{2}\|u+w-u\|^{2}=\frac{1}{2}\|u\|^{2}+(u, w-u)+\frac{1}{2}\|w-u\|^{2} .
$$

Hence $I_{1}$ is differentiable at $u$, with $I_{1}^{\prime}[u]=u$. Consequently, $I_{1}$ is Lipschitz continuous on bounded subsets of $H_{0}$.

Now we examine the term $I_{2}$. For $v, w \in H_{0}$, we define

$$
\langle v, w\rangle=\int_{\bar{S}} v(x) w(x) d_{\omega} x .
$$

By Riesz Representation Theorem, for each $v \in H_{0}$ there exists $v^{*} \in H_{0}$ uniquely such that

$$
\langle v, w\rangle=\left(v^{*}, w\right) \quad \forall w \in H_{0},
$$

i.e.

$$
\int_{\bar{S}} v(x) w(x) d_{\omega} x=\frac{1}{2} \int_{\bar{S}} \nabla v^{*}(x) \cdot \nabla w(x) d_{\omega} x .
$$

We will write $v^{*}=K v$.

We now demonstrate that if $u \in H_{0}$, then $I_{2}^{\prime}[u]=K[f(u)]$. To see this, note first that

$$
F(a+b)=F(a)+f(a) b+\left(\int_{0}^{1}(1-s) f^{\prime}(a+s b) d s\right) b^{2} .
$$

Thus for each $w \in H_{0}$,

$$
\begin{aligned}
I_{2}[w] & =\int_{\bar{S}} F(w) d_{\omega}=\int_{\bar{S}} F(u+w-u) d_{\omega} \\
& =\int_{\bar{S}} F(u)+f(u)(w-u) d_{\omega}+R \\
& =I_{2}[u]+\int_{\bar{S}} \nabla K[f(u)] \cdot \nabla(w-u) d_{\omega}+R,
\end{aligned}
$$

where the remainder term $R$ satisfies,

$$
|R| \leq C \int_{\bar{S}}\left(1+|u|^{p-1}+|w-u|^{p-1}\right)|w-u|^{2} d_{\omega}
$$




$$
\begin{aligned}
\leq C & \left(\int_{\bar{S}}|w-u|^{2}+|w-u|^{p+1} d_{\omega}\right) \\
& +C\left(\int_{\bar{S}}|u|^{p+1} d_{\omega}\right)^{\frac{p-1}{p+1}}\left(\int_{\bar{S}}|w-u|^{p+1} d_{\omega}\right)^{\frac{2}{p+1}} .
\end{aligned}
$$

By the equivalence of the norms on the same network, we have $R=o(\|w-u\|)$. Thus we see from Definition 3.1 and (3.5) that

$$
I_{2}[w]=I_{2}[u]+(K[f(u)], w-u)+o(\|w-u\|),
$$

as required.

By the equivalence of norms, for $\|u\|,\|\widetilde{u}\| \leq L$, we have

$$
\begin{aligned}
\left\|I_{2}^{\prime}[u]-I_{2}^{\prime}[\widetilde{u}]\right\|^{2} & =\|K[f(u)]-K[f(\widetilde{u})]\|^{2} \\
& =\int_{\bar{S}}|f(u)-f(\widetilde{u})|^{2} d_{\omega} \\
& \leq C \int_{\bar{S}}\left(\left(1+|u|^{p-1}+|\widetilde{u}|^{p-1}\right)|u-\widetilde{u}|\right)^{2} d_{\omega} \\
& \leq C(L)\|u-\widetilde{u}\|^{2},
\end{aligned}
$$

where we use mean value theorem on the third line of the inequalities. Thus $I_{2}^{\prime}$ is also Lipschitz continuous on bounded subsets of $H_{0}$.

Finally we have to verify the remaining hypotheses of the Mountain Pass Theorem. Clearly $I[0]=0$. Suppose that $u \in H_{0}$ with $\|u\|=r$ for $r>0$ to be selected below. Then

$$
I[u]=I_{1}[u]-I_{2}[u]=\frac{r^{2}}{2}-I_{2}[u] .
$$

Now hypothesis (3.2) implies that

$$
\left|I_{2}[u]\right| \leq C r^{p+1} .
$$

In view of (3.6), then

$$
I[u] \geq \frac{r^{2}}{2}-C r^{p+1} \geq \frac{r^{2}}{4}=: a>0,
$$

provided $r>0$ is small enough, since $p+1>2$. Now fix some nonzero element $u \in H_{0}$. Put $v:=t u$ for $t>0$ to be selected. Then

$$
\begin{aligned}
I[v] & =I_{1}[t u]-I_{2}[t u] \\
& =t^{2} I_{1}[t u]-\int_{\bar{S}} F(t u) d_{\omega} \\
& \leq t^{2} I_{1}[t u]-a t^{p+1} \int_{\bar{S}}|u|^{p+1} d_{\omega} \\
& <0
\end{aligned}
$$


for $t>0$ large enough.

We checked all the hypotheses of the Mountain Pass Theorem. There must consequently exist a nonzero function $u \in H_{0}$ with

$$
I^{\prime}[u]=u-K[f(u)]=0 .
$$

In particular for each $v \in H_{0}$, we have

$$
(u, v)=\frac{1}{2} \int_{\bar{S}} \nabla u \cdot \nabla v d_{\omega}=\int_{\bar{S}}-\Delta u v d_{\omega}
$$

and

$$
(K[f(u)], v)=\langle f(u), v\rangle=\int_{\bar{S}} f(u) v d_{\omega}
$$

therefore

$$
\int_{\bar{S}}-\Delta u v d_{\omega}=\int_{\bar{S}} f(u) v d_{\omega} .
$$

Let $T$ be a subset of vertices in $S$ and

$$
\chi_{T}(x)=\left\{\begin{array}{l}
1, x \in T \\
0, \text { otherwise. }
\end{array}\right.
$$

Then $\chi_{T} \in H_{0}$ since $\chi_{T}=0$ on $\partial S$. Taking $v=\chi_{T}$ where $T=\{x\}, x \in S$, we obtain

$$
-\Delta u(x)=f(u(x))
$$

Thus $u$ is a solution of (3.1).

Remark. In the continuous case, since the Sobolev embedding is necessary in the proof, we need the condition on $p$, such as $1<p<\frac{n+2}{n-2}$, where $n(\geq 3)$ is a space dimension. But in the discrete case, we use the equivalence of the norms defined on finite dimensional space, we do not need the upper bound of $p$.

Remark. For the case $p=1$, if we consider a simple network with standard weight (Fig 1). We set $\partial S=\{a, d\}$ and $S=\{b, c\}$. Consider the equation

$$
\left\{\begin{array}{l}
-\Delta u(x)=u(x), \quad x \in S, \\
u(x)=0, \quad x \in \partial S .
\end{array}\right.
$$




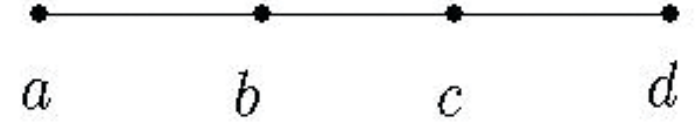

Figure 1. The standard weighted network with $S=\{b, c\}$ and $\partial S=\{a, d\}$

Which means that

$$
\begin{aligned}
u(a) & =0 \\
\frac{1}{2}(u(b)-u(a))+\frac{1}{2}(u(b)-u(c)) & =u(b) \\
\frac{1}{2}(u(c)-u(d))+\frac{1}{2}(u(c)-u(b)) & =u(c) \\
u(d) & =0 .
\end{aligned}
$$

Solving the above system, we have $u(a)=u(b)=u(c)=u(d)=0$. The trivial solution is the only solution.

So the condition $p>1$ is optimal.

\section{§4. Existence and Uniqueness of the Solution Case II}

In this section we will consider the following Dirichlet boundary value problem :

$$
\left\{\begin{array}{l}
-\Delta u(x)=f(x, u(x)), \quad x \in S, \\
u(x)=\sigma(x), \quad x \in \partial S .
\end{array}\right.
$$

We assume that the function $f$ is bounded and nonincreasing with respect to the second variable.

We will show the existence of a solution using the solvability of the Dirichlet boundary value problem for Poisson equation ([1]).

First we define a mapping $A: H \rightarrow H$ as following: For given $u \in H$, let $w \in X$ be the solution of linear problem 


$$
\left\{\begin{array}{l}
-\Delta w(x)=f(x, u(x)), \quad x \in S \\
w(x)=\sigma(x), \quad x \in \partial S .
\end{array}\right.
$$

We write $A[u]=w$ whenever $w$ is derived from $u$ via (4.2). Since the Dirichlet boundary value problem (4.2) has a unique solution([1]), the mapping $A$ is well-defined.

If the mapping $A$ has a fixed point, then the fixed point is a solution of (4.1).

Now we state the Schaefer's Fixed Point Theorem (For more details and proof of the theorem, see [8]).

Theorem 4.1 (Schaefer's Fixed Point Theorem). Suppose

$$
A: X \rightarrow X
$$

is a continuous and compact mapping. Assume further that the set

$$
M=\{u \in X \mid u=\lambda A[u] \text { for some } 0 \leq \lambda \leq 1\}
$$

is bounded. Then A has a fixed point.

By Schaefer's Fixed Point Theorem, we can show the existence of a solution and we need the maximum principle for elliptic operator on network to show the uniqueness of the solution.

Lemma 4.1. Let $Q[\cdot]=\Delta[\cdot]+f(\cdot)$, where $f$ is non-increasing function. Then for $u, v: \bar{S} \rightarrow \mathbf{R}$,

(i) if $Q u \leq Q v$ in $S$ and $u \geq v$ on $\partial S$, Then $u \geq v$ in $S$.

(ii) if $Q u \geq Q v$ in $S$ and $u \leq v$ on $\partial S$, Then $u \leq v$ in $S$.

(iii) if $Q u=Q v$ in $S$ and $u=v$ on $\partial S$, Then $u=v$ in $S$.

Proof. We only show (i). The others can be derived similarly.

Let

$$
h(x):=u(x)-v(x)
$$

and $h$ attains its minimum at $x_{0}$. It suffices to show that $h\left(x_{0}\right) \geq 0$. Suppose that $h\left(x_{0}\right)<0$. Notice that $x_{0} \in S$, since $h(x) \geq 0$ on $\partial S$. By our assumption,

$$
h\left(x_{0}\right) \leq \sum_{y \in \bar{S} \backslash\left\{x_{0}\right\}} h(y) \frac{\omega x_{0}, y}{d_{\omega} x_{0}}, \quad \text { i.e., } \quad \Delta h\left(x_{0}\right) \geq 0 .
$$


Since $f(x, s)$ is non-decreasing in the variable $s$, we have

$$
0 \geq(Q u-Q v)\left(x_{0}\right)=f\left(x_{0}, u\left(x_{0}\right)\right)-f\left(x_{0}, v\left(x_{0}\right)\right)+\Delta h\left(x_{0}\right) \geq 0
$$

and hence

$$
f\left(x_{0}, u\left(x_{0}\right)\right)=f\left(x_{0}, v\left(x_{0}\right)\right) \text { and } \Delta h\left(x_{0}\right)=0 .
$$

Since $h$ attains its minimum at $x_{0}$, we must have $h(x)=h\left(x_{0}\right)$ for every $x \sim x_{0}$. Now for any $z \in \partial S$, there exists a path

$$
x_{0} \sim x_{1} \sim x_{2} \sim \cdots \sim x_{n-1} \sim x_{n}=z,
$$

since $S$ is connected. By applying the same argument as above inductively, we see that $h(z)=h\left(x_{0}\right)<0$, which is a contradiction. Thus we have $h\left(x_{0}\right) \geq 0$.

Now we are ready to show the main result of this section.

Theorem 4.2. $\quad$ Nonlinear poisson equation on network (4.1) has a unique solution.

Proof. First we show the existence of a solution by the Schaefer's Fixed Point Theorem.

We can show that $A: X \rightarrow X$ is continuous and compact by the explicit formula of the solution of Poisson equation ([1]).

Now we will show that the set

$$
M=\{u \in X \mid u=\lambda A[u] \text { for some } 0 \leq \lambda \leq 1\}
$$

is bounded.

Assume $u \in X$,

$$
u=\lambda A[u] \quad \text { for some } 0 \leq \lambda \leq 1 .
$$

Then $\frac{u}{\lambda}=A[u]$, i.e.

$$
\left\{\begin{array}{l}
-\Delta u(x)=\lambda f(x, u(x)), \quad x \in S, \\
u(x)=\lambda \sigma, \quad x \in \partial S .
\end{array}\right.
$$

Consider the norm of $u$ 


$$
\begin{aligned}
\|u\|^{2} & =\frac{1}{2} \int_{\bar{S}}|\nabla u(x)|^{2} d_{\omega} x+\lambda \int_{\partial S}|u(z)|^{2} d_{\omega} z \\
& =\int_{\bar{S}} \Delta u u d_{\omega}+\lambda\|\sigma\|_{2}^{2} \\
& =\int_{S} \Delta u u d_{\omega}+\int_{\partial S} \Delta u u d_{\omega}+\lambda\|\sigma\|_{2}^{2} \\
& =\int_{S} \lambda f(x, u(x)) u(x) d_{\omega} x+\int_{\partial S} \lambda \frac{\partial u}{\partial n}(z) \sigma(z) d_{\omega} z+\lambda\|\sigma\|_{2}^{2} \\
& \leq \int_{S}|f(x, u(x)) u(x)| d_{\omega} x+\int_{\partial S}\left|\frac{\partial u}{\partial n}(z) \sigma(z)\right| d_{\omega} z+\|\sigma\|_{2}^{2} .
\end{aligned}
$$

Then we have

$$
\begin{gathered}
\int_{S}|f(x, u(x)) u(x)| d_{\omega} x \leq\|f\|_{\infty}\|u\|_{1}, \\
\int_{\partial S}\left|\frac{\partial u}{\partial n}(z) \sigma(z)\right| d_{\omega} z \leq|\partial S|\left(\|u\|_{\infty}+\|\sigma\|_{\infty}\right)\|\sigma\|_{\infty} .
\end{gathered}
$$

Since $f$ is bounded, $\sigma$ is given and all the norms on the same network are equivalent, we have

$$
\|u\|^{2} \leq C_{1}\|u\|+C_{2}, \quad \text { for some } C_{1}, C_{2} \geq 0 .
$$

Finally, we have

$$
\|u\| \leq \frac{C_{1}+\sqrt{C_{1}^{2}+4 C_{2}}}{2}<\infty .
$$

So $A$ satisfies every hypothesis of Schaefer's Fixed Point Theorem, we have $\exists u \in X$ such that $A[u]=u$ which is a solution of (4.1).

The uniqueness can be shown by Lemma 4.1 (iii).

Remark. From the proof of the theorem we obtain that :

(a) the boundedness of the function $f$ implies the existence of a solution for the Dirichlet boundary value problem (4.1),

(b) the nonincreasing condition implies the uniqueness of a solution. 


\section{References}

[1] S.-Y. Chung and C. A. Berenstein, $\omega$-harmonic functions and inverse conductivity problems on networks, SIAM J. Appl. Math. 65 (2005), no. 4, 1200-1226 (electronic).

[2] F. R. K. Chung, Spectral graph theory, Published for the Conference Board of the Mathematical Sciences, Washington, DC, 1997.

[3] Discrete Green's functions, J. Comb. Theory(A), 91 (2001), 191-214.

[4] F. R. K. Chung and K. Oden, Weighted graph Laplacians and isoperimetric inequalities, Pacific J. Math. 192 (2000), no. 2, 257-273.

[5] D. M. Cvetković, M. Doob and H. Sachs, Spectra of graphs, Academic Press, New York, 1980.

[6] E. B. Curtis and J. A. Morrow, The Dirichlet to Neumann map for a resistor network, SIAM J. Appl. Math. 51 (1991), no. 4, 1011-1029.

[7] L. C. Evans, Partial Differential Equations, Grad. Stud. Math. Vol 19.

[8] D. Gilbarg and N. S. Trudinger, Elliptic partial differential equations of second order, Second edition, Springer, Berlin, 1983.

[9] A. Murakami and M. Yamasaki, Nonlinear potentials on an infinite network, Mem. Fac. Sci. Shimane Univ. 26 (1992), 15-28.

[10] M. Yamasaki, Nonlinear Poisson equations on an infinite network, Mem. Fac. Sci. Shimane Univ. 23 (1989), 1-9. 